

\title{
A never ending frontier
}

In the supplement accompanying this issue of Nature Structural Biology, the extraordinary advances during the last few years in structural characterization by nuclear magnetic resonance spectroscopy (NMR) are reviewed'. There are, inevitably, triumphs and disappointments. The anticipated details of dynamics and of the ionization states of groups in proteins and nucleic acids are now forthcoming. The precision and accuracy of solution structures by NMR continues to improve and the upper range of molecular weights accessible to NMR structure determination creeps ever upwards. Indeed, it is now possible for NMR structural biologists to give a lecture to a general audience without having to apologize for not being crystallographers. Very recently, structural refinement involving new information, the anisotropies to hydrodynamic and magnetic susceptibility axes, provides an exceptional stimulation to a possible completely fresh approach to NMR structure determination, perhaps in some much larger macromolecules. All these provide the NMR structural biologists with much to look forward to.

Why are there disappointments? With at least one major trend in structural biology being the determination of ever larger macromolecular complexes (the GroEL-GroES folding machine being the most recent example ${ }^{2}$ ) the size restriction that NMR spectroscopy imposes must be regarded as a significant limitation of its utility compared to crystallography or electron microscopy. On the other hand, for smaller proteins and nucleic acid molecules, once reasonable solution conditions are found, NMR structure determination is largely predictable, that is, deterministic. This encouraged an earlier optimism that NMR would be highly competitive with crystallography for biological macromolecules of up to $\sim 15,000 M_{\mathrm{r}}$. While this has turned out to be partly

nature Structural biology

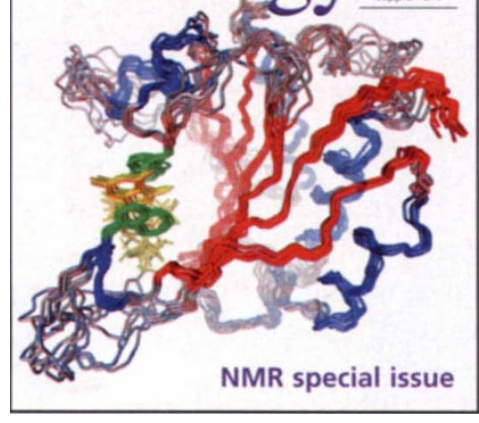
true, the maturity of X-ray crystallography should not have been confused with technical quiescence, and the productivity of crystallography has been dramatically improved by a large number of technical advances, so that the potential advantages of NMR have not been as generally evident as they might.

A further issue has been that, in spite of the elegance of Richard Ernst's approach to multidimensional $\mathrm{NMR}^{3}$, most of the developments in NMR's application to biopolymers ${ }^{4,5}$ have been of an incremental and highly technical nature, which have not been accorded much recognition by the general scientific audience. Many physicists, for example, take the view that nothing is new in NMR since Abragam's The Principles of Nuclear Magnetism published in $1961^{6}$ (which will remain an Aristotelian classic in style and content), but this view is too narrow, because it ignores how those principles are applied in practice to a range of problems in biology and chemistry. Using the physical and chemical properties of nuclear spins in biomolecular structural studies has required exceptional efforts integrating chemical physics, instrumentation development, new algorithms, and labelling strategies. 
1. NMR Supplement Nature Struct. Biol. 4 . 841-865 (1997)

2. Xu, Z., Horwich, A. \& Sigler, P.B. Nature 388, 741-750 (1997)

3. Ernst, R.R. Ang. Chem. Int. Ed. 31, 805-823 (1992).

4. Wuthrich, K. NMR of proteins and nucleic acids (John Wiley and Sons, New York: 1986).

5. Cavanagh, J., Fairbrother, W.J., Palmer III, A.G. \& Skelton, N.J. Protein NMR Spectroscopy (Academic Press, San Diego; 1996).

6. Abragam, A. The Principles of Nuclear Magnetism (Clarendon Press, Oxford; 1961). 7. Shuker, S.B., Hajduk, P.J., Meadows, R.P. \& Fesik, S.W. Science 274, 1531-4 (1996).

David Cowburn is at the Rockefeller University, Box 163, 1230 York Avenue, New York, New York 10012-6399, USA. email: cowburn@rockefeller.edu
What can NMR now do, and do well, in structural biology? Two significant areas of research immediately spring to mind — the detailed understanding of intermolecular interactions, and functional genomics - where NMR should play a leading role. NMR can provide a realistic, dynamic and/or time averaged view of a macromolecule-ligand interface (or, indeed, the transient intramolecular interfaces seen during protein folding) not available by other methods. This is an advantage in probing the role of local entropic contributions to binding using relaxation methods, and in the dissection of localized weak contributions, as in the 'SAR (structure activity relationships) by NMR' approach? NMR also provides an invaluable reality check on the exuberant interpretation of crystal packing interactions as biological significant phenomena, occasionally practiced by even some of the best crystallographers. These advantages are restricted by the limited precision of NMR structures compared to the very high resolution crystallographic structures, and by the tyranny of slow molecular rotation at increasing molecular weight.

With regard to functional genomics, it is clear that: (i) there are a limited number of protein fold types; (ii) many intracellular proteins are made up of combinations of modular domains, many individually folding entities; and (iii), that although many intermolecular interactions (especially protein-protein) occupy large surface areas, the driving forces for these can be mimicked by significantly smaller surface contact areas. Especially in signal transduction and transcriptional control, these observations suggest that a significant number of new gene sequences can have their protein products reduced to a manageable size for NMR-based structural investigation, and their ligands mimicked, based on structural results, for general investigational and potential therapeutic uses, possibly even prior to any characterization of the gene product in vivo. Such a development would be expected to have substantial impact on understanding the associated cellular pathways, and interactions. This approach - the integration of structural studies with bioinformatics and the use of ligand design from new chemical approaches - is in contrast to scaling up, in an industrial fashion, the current use of structural biology/NMR, and incrementally improving the processes of solution structural determination, as the final stages of characterizing a gene product.

How can these ideas become a more practical reality? At every stage of the last 20 years of NMR applications, increased field strengths have provided additional sensitivity and resolution, and stimulated innovations. New applications of the measurements of the effects of partial orientation dependent of magnetic anisotropy will benefit similarly, or more, by use of increased field strengths. Unfortunately, there are few new superconducting alloys with the requisite properties for big jumps in field strength, and the interest in developing them has declined with the advent and excitement of high temperature superconductors presenting new scientific challenges. A $1 \mathrm{GHz}$ or higher field NMR system is much wished for, but is not in any likely five-year plan.

With such a capital-intensive technique as NMR, there is naturally suspicion that a few ultra-high field magnet centres would encroach too egregiously on the funds that otherwise might be available for individual or institutional resources. But realistically, the development of new applications and understanding how they can be best applied in structural biology is now seriously hampered by the limited access to ultra high field resources, and the development of new ones is not accorded high priority. The argument is frequently made that other (more immediately pressing) issues exist, and that the associated sensitivity and resolution increments are too expensive. The crystallographic community did not take the equivalent position with regard to the provision of bright $\mathrm{X}$-ray sources and they are now the fortunate beneficiaries of synchrotron radiation sources, paid for by central funds with limited encroachment of individual grants. The case for a significant increase in the number of state of the art $(800 \mathrm{MHz})$ systems and development of the feasible, but engineering costly $900 \mathrm{MHz}$ systems at $21 \mathrm{~T}$ is strong, for the purposes of advancing the development of these promising applications of NMR in structural biology.

Nevertheless, the flood of new ideas and developments in this field continues apace. For a quieter life, NMR-structural biologists might wish for a boundary condition to be reached, but more realistically, this area continues at the edge of a never ending frontier. 